\title{
Understanding the Hydrogen and Oxygen Gas Pressure Dependence of the Tribological Properties of Silicon Oxide-Doped Hydrogenated Amorphous Carbon Coatings
}

\author{
K. Koshigan ${ }^{1, \dagger}$, F. Mangolini' ${ }^{1, \dagger}$, J.B. McClimon ${ }^{2}$, B. $\operatorname{Vacher}^{1}$, S. Bec ${ }^{1}$, R.W. \\ Carpick $^{3}$, and J. Fontaine ${ }^{1, *}$
}

1 Laboratoire de Tribologie et Dynamique des Systèmes, Ecole Centrale de Lyon, CNRS UMR 5513, Université de Lyon, 69134, Ecully cedex, France

2 Department of Materials Science and Engineering, University of Pennsylvania, Philadelphia, Pennsylvania 19104, USA

3 Department of Mechanical Engineering and Applied Mechanics, University of Pennsylvania, Philadelphia, Pennsylvania 19104, USA

$\dagger \quad$ Equally contributing authors

Short Title: Tribochemistry of Silicon Oxide-Doped Diamond-Like Carbon

* To whom correspondence should be addressed: julien.fontaine@ec-lyon.fr 


\section{Abstract}

Silicon oxide-doped hydrogenated amorphous carbons (a-C:H:Si:O) are amorphous thin films used as solid lubricants in a range of commercial applications, thanks to its increased stability in extreme environments, relative to amorphous hydrogenated carbons (a-C:H). This work aims to develop a fundamental understanding of the environmental impact on the tribology of a-C:H:Si:O. Upon sliding an a-C:H:Si:O film against a steel counterbody, two friction regimes develop: high friction in high-vacuum and low gas pressure (oxygen pressure $<10$ mbar; hydrogen pressure $<50$ mbar), and a low friction regime at higher gas pressures (10 mbar < oxygen pressure $<500$ mbar; 50 mbar < hydrogen pressure $<1000$ mbar;). Scanning electron microscopy (SEM) revealed that the tribological behavior of a-C:H:Si:O is governed by adhesive junctions at the sliding interface. At low gas pressures, material transfer from the steel pin to the a-C:H:Si:O flat occurs. At higher gas pressures, a tribofilm forms on the steel countersurface. Raman and near edge X-ray absorption spectroscopy (NEXAFS) spectroscopies demonstrate that upon sliding under the higher gas pressure, low friction regime, a surface layer with an elevated fraction of $\mathrm{sp}^{2}$-bonded carbon atoms forms. These changes indicate that these gases favor the release of the adhesive junctions by dissociatively reacting with the mechanically-stressed $\mathrm{sp}^{2}$ carbon-rich surface layer. 


\section{Introduction}

Amorphous hydrogenated carbon $(\mathrm{a}-\mathrm{C}: \mathrm{H})$ films are metastable carbon-based coatings used in a wide range of technological applications thanks to their impressive properties, notably their strength, ability to withstand many harsh physical and chemical environments, and their ability to form continuous, conformal, smooth coatings ${ }^{1}$. In light of their outstanding tribological properties, namely low friction combined with high wear resistance, $\mathrm{a}-\mathrm{C}: \mathrm{H}$ films have been used as protective coatings in cutting tools, hard disks, car engines, and microelectromechanical systems (MEMS) ${ }^{2}$. Furthermore, their chemical inertness, corrosion resistance, and biocompatibility make them good candidates for biomedical applications ${ }^{3,4}$. Nevertheless, these coatings do have limitations, namely high residual stress (more than $6 \mathrm{GPa}$ for some amorphous carbon films ${ }^{5}$ ), which can lead to delamination of the coating from its substrate, low thermal stability, and friction which varies with the sliding environment.

To impart new properties or to modify existing ones while keeping the characteristic amorphous structure of a-C:H films, different elements such as $\mathrm{Si}, \mathrm{O}, \mathrm{F}, \mathrm{N}$, and metals ${ }^{6-8}$ can be incorporated into the amorphous carbon network. It has also been shown by Panwar et al. ${ }^{9}$ that the incorporation of boron and phosphorous inside a tetrahedral amorphous carbon network decreases the $\mathrm{sp}^{3}$ fraction in the coating.

The incorporation of oxygen together with silicon in a-C:H films leads to the development of a new class of coatings, i.e., silicon oxide-doped hydrogenated amorphous carbon (a-C:H:Si:O), sometimes referred to as diamond-like nanocomposite (DLN) or SiOdoped diamondlike carbon. a-C:H:Si:O films are so far generally described as a fully amorphous film consisting of two interpenetrating and interbonded networks, one being a silica glass $\left(\mathrm{SiO}_{\mathrm{x}}\right)$ network and the second one, an amorphous hydrogenated carbon network $(\mathrm{a}-\mathrm{C}: \mathrm{H})$. This structure is claimed to exhibit lower internal stress (typically less than $1 \mathrm{GPa}$ ${ }^{10}$ ) than a:C-H, leading to good adhesion with various substrates. The drop in the compressive residual stress in a-C:H:Si:O films has been ascribed by Neerinck et al. to the presence of Si$\mathrm{C}$ bonds, which are longer that $\mathrm{C}-\mathrm{C}$ bonds ( $\mathrm{Si}-\mathrm{C}$ bond length $=1.89 \AA$ vs. $\mathrm{C}-\mathrm{C}$ bond length $=$ $1.54 \AA)^{11}$.

In addition, the thermal stability of a-C:H:Si:O films has been found to be higher than that of a-C:H. This is suggested to arise from the fourfold coordination of Si atoms stabilizing the carbon atoms in the $\mathrm{sp}^{3}$ hybridization state, thus inhibiting their conversion into threefoldcoordinated $\mathrm{sp}^{2}$ carbon at high temperature. This would imply that the activation energy required for the conversion of $\mathrm{sp}^{3}$-hydridized carbon atoms into $\mathrm{sp}^{2}$ in a-C:H:Si:O is higher 
than in a-C:H, which has been estimated by Mangolini et al. ${ }^{12}$ to be $3.5 \pm 0.5 \mathrm{eV}$. However, no experimental evidence to explain the effect of the incorporation of silicon and oxygen in the amorphous carbon matrix on the thermal stability of the resulting structure has even been reported.

Concerning the tribological behaviour of Si-doped DLCs, it has been shown that incorporation of $\mathrm{Si}$ reduces the extent to which the frictional properties of DLCs are dependent on the environmental conditions ${ }^{13,14}$. In particular, Gilmore and Hauert demonstrated that the incorporation of approximately 4 at. \% of Si into the amorphous carbon network can render its friction response less dependent on the sliding environment ${ }^{15}$ while sliding against steel. More recently, Scharf et al. ${ }^{16}$ demonstrated that the friction mechanism of a-C:H:Si:O is mainly governed by the interfacial sliding between the a-C:H:Si:O coating and the friction-induced tribofilm formed on the silicon nitride or steel countersurface. Moreover, the environment plays a major role on the composition and mechanical properties of this interfacial film: while in dry environment the interfacial film mainly consists of carbon and has a low shear strength (9 GPa), under humid conditions the interfacial film consists of silica fragments and exhibits a high shear strength $(78 \mathrm{GPa})$.

Even though the role of water vapour in the friction behaviour of a-C:H:Si:O has been examined multiple times, no work focusing on the effect of oxygen and hydrogen gas on the tribological response of a-C:H:Si:O-vs.-steel tribosystems has ever been published in the literature.

This study aims to shed light on the origin of the environmental dependence of the tribological properties of a-C:H:Si:O films sliding against steel. Tribological tests were conducted under different environmental conditions, ranging from high vacuum to elevated gas pressures of oxygen and hydrogen. The subsequent characterization of both the a$\mathrm{C}: \mathrm{H}: \mathrm{Si}: \mathrm{O}$ coatings and the steel countersurface by multiple analytical techniques (interferometry, SEM/EDX, Raman and NEXAFS) allowed elucidating the structural transformations and chemical reactions that occurred upon sliding, and understanding how they affect the environmental dependence of the friction force. 


\section{Experimental}

\subsection{Materials}

Silicon oxide-doped hydrogenated amorphous carbon (a-C:H:Si:O) coatings were deposited on silicon wafers by Sulzer-Metco Inc. (Winterthur, Switzerland) using a proprietary plasma-enhanced chemical vapor deposition (PECVD) process, whose details are described elsewhere ${ }^{11,16-18}$. The thickness of the a-C:H:Si:O coating was approximately 2 $\mu \mathrm{m}$.

A detailed description of the characterization of bulk and surface chemistry as well as nanomechanical properties of the as-received coatings will be the subject of a separate publication (the composition, surface roughness, and mechanical properties of the asdeposited material are provided in the Supporting Information).

\subsection{Methods}

\subsubsection{Tribological Tests under Controlled Environment}

Friction experiments were performed with a linear reciprocating pin-on-flat tribometer placed inside an high vacuum (HV) chamber and allowing gas introduction, which has been extensively described previously ${ }^{19}$. The calibration of the optical sensors was performed using standard weights for the normal force and a pulley for the tangential force.

The friction coefficient is calculated as the ratio of the tangential force and the applied normal load. A time-averaging data-reduction method is applied for processing the friction data by averaging the data acquired during one sliding cycle. To avoid end-point effects, only the data corresponding to a slider position between $1 / 8$ and $7 / 8$ of the stroke length were considered. The standard deviation of the values acquired during this period is also evaluated.

Leaking different gases into the tribometer chamber is achieved by means of a standard leak valve. The base pressure in the chamber is lower than $10^{-7}$ mbar. A Baratron process manometer controls the gas pressure and a differential mass spectrometer (Transpector 2 Gas Analysis System, Inficon, NY, USA) controls the gas purity in the chamber.

Tribological tests were performed at room temperature using 52100 steel pins with a radius of curvature of $8 \mathrm{~mm}$. Before each experiment, the pins were mechanically polished leading to a root-mean square roughness of $27.3 \pm 0.2 \mathrm{~nm}$ (measured by white light interferometry) and then sonicated in acetone and isopropanol. Once introduced in the vacuum chamber, the high vacuum is obtained through a turbo pump without any baking. 
Tribotests were run under an applied load of $3 \mathrm{~N}$ (corresponding to an initial maximum Hertzian contact stress of $430 \mathrm{MPa}$ and an apparent contact radius of $60 \mu \mathrm{m}$ ). The sliding speed and stroke length were $2 \mathrm{~mm} / \mathrm{s}$ and $2 \mathrm{~mm}$, respectively. At least three independent tribological tests were performed for each environmental condition to check reproducibility.

\subsubsection{Analysis of a-C:H:Si:O after Tribotesting}

The wear tracks and the contact region on the pin were analysed by scanning electron microscopy (ESEM-FEG XL30, FEI-Philips, UK) with energy dispersive X-ray spectroscopy (EDX, SDD Xmax $50 \mathrm{~mm}^{2}$, Oxford Instruments, UK). The EDX analyses were performed using a $5 \mathrm{kV}$ acceleration voltage.

A Zygo NewView ${ }^{\mathrm{TM}} 6300$ scanning white-light interferometer (SWLI) (Zygo Corporation, Middlefield, CT, USA) was used for characterizing the wear rate of the a$\mathrm{C}: \mathrm{H}: \mathrm{Si}$ :O films. All measurements were performed with a $20 \mathrm{X}$ objective (vertical resolution $<1 \mathrm{~nm}$ ). A Matlab ${ }^{\circledR}$ (MathWorks ${ }^{\circledR}$, Natick, MA) script was developed for processing the profilometry data. To determine the worn volume, the images were first leveled and then the volume removed upon sliding was computed.

Raman spectra were acquired using a near-field scanning Raman spectrometer (NTMDT NTGRA ${ }^{\mathrm{TM}}$ Spectra Upright, NT-MDT Co., Moscow, Russia) with an excitation wavelength of $532 \mathrm{~nm}$. A detailed description of the spectrometer is provided in the Supporting Information. In the present work, all Raman spectra were recorded with a laser power of $60 \mu \mathrm{W}$ to avoid any damage and within a spectral range of $278-2676 \mathrm{~cm}^{-1}$. A 600 lines $/ \mathrm{mm}$ grating provided a spectral resolution better than $2 \mathrm{~cm}^{-1}$. All the Raman spectra presented in the paper are reported without any correction, i.e., as-acquired. Raman spectroscopic measurements were carried out on several points to check lateral homogeneity.

Imaging near-edge X-ray absorption fine structure (NEXAFS) spectroscopic measurements were performed using the parallel processing imaging system at the NIST/Dow endstation of beamline U7A at the National Synchrotron Light Source (NSLS), Brookhaven National Laboratory (Upton, NY, USA). This imaging technique, which allows for the structural and chemical characterization of the near-surface region with a lateral resolution of $50 \mu \mathrm{m}$ and a field of view of $18 \times 13 \mathrm{~mm}^{2}$, has been extensively described earlier $^{20}$. The photon source for this beamline is a bending magnet, and covers an energy range from 100 to $800 \mathrm{eV}$. The photon flux is $2 \times 10^{11}$ photons/second/ $0.1 \%$ bandwidth. In the present work, NEXAFS images were acquired in partial electron yield (PEY) mode using a photon energy range of 260-340 eV for the carbon K-edge (energy resolution of $0.1 \mathrm{eV}$ ). The 
entrance grid bias (EGB) of the channeltron detector was set to 50, 150, and $220 \mathrm{~V}$ for the carbon K-edge. Changing the EGB voltage allows NEXAFS measurements with different surface sensitivity to be performed ${ }^{21}$. The monochromator energy was calibrated using the carbon K-edge $\pi^{*}$ transition of freshly-cleaved highly ordered pyrolytic graphite (HOPG, grade 2, SPI Supplies, West Chester, PA, USA), located at $285.5 \mathrm{eV}$. All NEXAFS images were first normalized to the absorption current measured simultaneously from a gold mesh placed in the beamline upstream from the analysis chamber, and then normalized to the spectral intensity in the pre-edge region (intensity between 270 and $280 \mathrm{eV}$ ).

\section{Results}

\subsection{Friction measurements}

Figure 1a displays the evolution of the coefficient of friction as a function of the number of reciprocation cycles during tribological tests performed on the a-C:H:Si:O film under different environmental conditions, ranging from high vacuum (HV) to elevated pressures of hydrogen or oxygen. Under HV and low gas pressure (i.e., < 10 mbar, independent of the gas introduced in the tribometer chamber), the friction forces increase rapidly with the number of cycles, thus resulting in a very high coefficient of friction $(1.2 \pm 0.2)$ after only 15 sliding cycles. For oxygen and hydrogen pressures above 10 mbar and 50 mbar, respectively, a drastic reduction in friction, by a factor of 50 and 20 for oxygen and hydrogen respectively, was observed after a running-in period (approximately 50 sliding cycles).

Figure $1 \mathrm{~b}$ displays the dependence of the average coefficient of friction with the corresponding standard deviation (calculated considering the force value at steady-state, i.e., for the last 400 sliding passes on the test as a function of the pressure in the tribometer chamber; for the case of the tests performed under HV or for gas pressures less than 10 mbar, the average coefficient of friction with the corresponding standard deviation was calculated considering all data points). Upon increasing the oxygen pressure in the chamber, the friction coefficient decreases to its lowest value of $0.02 \pm 0.01$ between 12 and 14 mbar of oxygen and then slightly increases to reach $0.06 \pm 0.01$ for a maximum gas pressure of 500 mbar. In the case of hydrogen, the transition between high friction and low friction coefficient occurred between 10 and 50 mbar. Upon further increasing the hydrogen pressure, the friction coefficient kept on decreasing and reached its lowest value $(0.02 \pm 0.01)$ at 1000 mbar. 
(a)

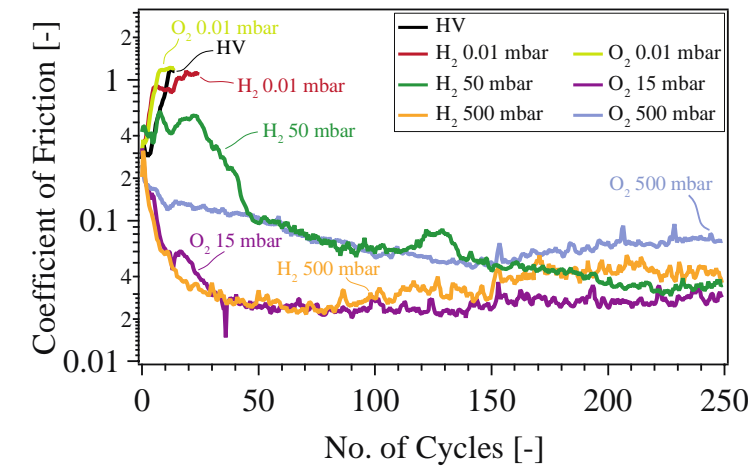

(b)

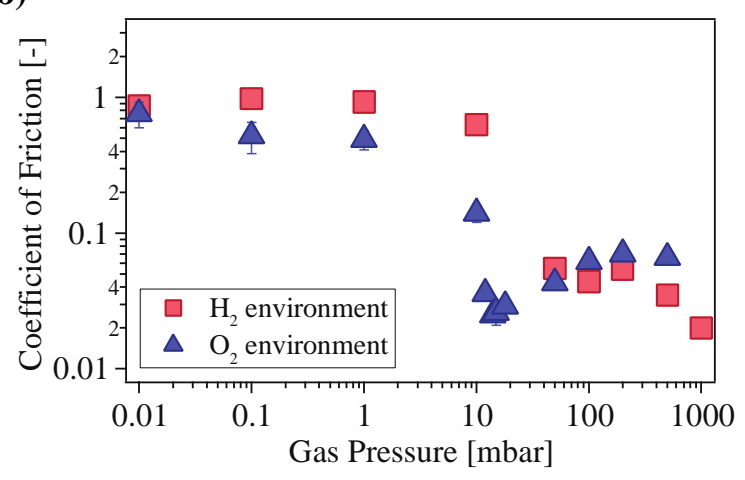

Figure.1. a) Coefficient of friction versus number of cycles during tribological tests (normal load: $3 \mathrm{~N}$; sliding speed: $2 \mathrm{~mm} / \mathrm{s}$; stroke: $2 \mathrm{~mm}$ ) performed on a-C:H:Si:O using a steel pin under different environmental conditions (from high vacuum to different oxygen and hydrogen pressures); b) the computed steady-state coefficient of friction is displayed as a function of the oxygen and hydrogen pressure.

Figure 2 shows a SEM micrograph of the heavily worn region of a-C:H:Si:O film after tribotesting under a low pressure of oxygen $(0.01 \mathrm{mbar})$. The presence of iron on a$\mathrm{C}: \mathrm{H}: \mathrm{Si}: \mathrm{O}$, which was substantiated by EDX analyses (not shown), clearly indicates the preferential transfer of materials from the steel pin to the a-C:H:Si:O flat under $\mathrm{HV}$ and at low gas pressure ( $<10 \mathrm{mbar}$ of oxygen and $<50$ mbar of hydrogen). Very similar results were seen for sliding under HV and a low pressure of hydrogen (0.01 mbar).

The EDX analysis on the pin used for running tribological tests at 100 mbar of oxygen (Figure 2e) indicates that the tribofilm formed on the pin is constituted of carbon, oxygen and silicon, thus suggesting a transfer from the a-C:H:Si:O flat to the steel pin. The formation of tribofilms composed of carbon, oxygen, and silicon on steel pins was also observed for tribotests carried out at gas pressures higher than 15 mbar of oxygen and 100 mbar of hydrogen. Therefore, we conclude that the transfer of iron to the flat ceases upon increasing the gas pressure in the chamber to between 10 and 14 mbar of oxygen, and 
between 10 and 50 mbar of hydrogen, i.e., the same oxygen and hydrogen pressures at which a drastic reduction in friction was observed.
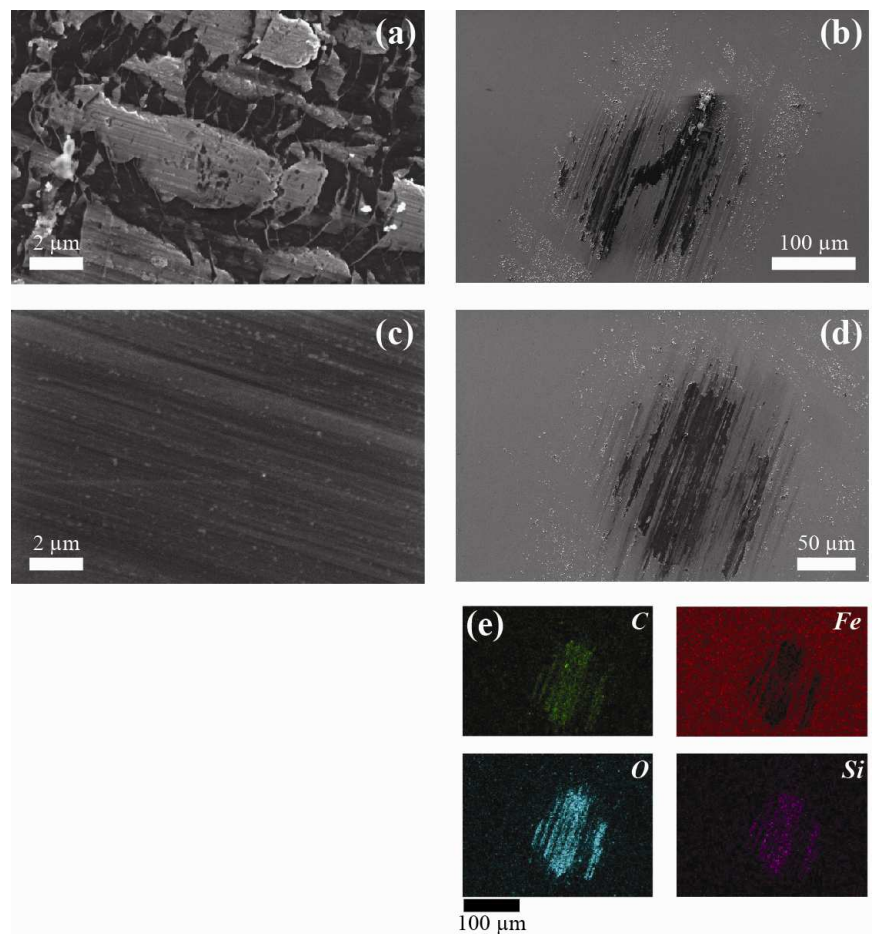

Figure.2. SEM micrographs of a-C:H:Si:O after tribological testing at 0.01 mbar (a) and 100 mbar of oxygen (c). The SEM micrographs of the steel pins used for running the tests at 0.01 mbar of oxygen and at 100 mbar of oxygen are displayed in (b) and (d), respectively; (e) EDX analysis of the steel pin employed for the experiment run at 100 mbar of oxygen.

To gain insights into the structural changes occurring in a-C:H:Si:O upon sliding as well as into the structure of the tribofilm formed on the steel pin, Raman analyses were performed (Figure 3). The visible Raman spectrum of amorphous carbon-based materials is dominated by the signal originating from $\mathrm{sp}^{2}$-hybridized carbon, since the scattering cross section of $\pi$ states is more than 50 times higher than that of $\sigma$ states $^{22}$. The characteristic broad envelope of amorphous carbon-based materials is commonly interpreted as a convolution of two peaks, the $\mathrm{G}$ and $\mathrm{D}$ bands, superposed on a photoluminescence background signal. The $\mathrm{G}$ band near $1514 \mathrm{~cm}^{-1}$ corresponds to the in-plane bond stretching motions of pairs of $\mathrm{sp}^{2}$ atoms (both in rings and chains), whereas the D band near $1298 \mathrm{~cm}^{-1}$ is a breathing mode of $\mathrm{sp}^{2}$ atoms in six-fold aromatic rings, which only shows up when there are variations in the ring structure (the D peak is absent for large pristine graphene and graphite samples). As for the photoluminescence signal, Rose et al. ${ }^{23}$ demonstrated that the origin of the photoluminescence background in Raman spectra of amorphous carbon is 
twofold: it can be due to the presence of carbon-hydrogen bonds, or to the clustering of the $\mathrm{sp}^{2}$ phase.

The characteristic Raman spectrum of unworn a-C:H:Si:O (from the as-grown, noncontact region) exhibits the characteristic broad envelope for amorphous hydrogenated carbon-based materials (Figure. 3). Upon sliding under oxygen or hydrogen environments, no significant changes in the Raman spectra of a-C:H:Si:O were observed, regardless of the gas pressure.

Raman spectroscopic measurements were also performed on the wear particles created during the sliding process on the a-C:H:Si:O samples as well as on the tribofilm formed on steel pins. In both cases, the spectra exhibited clear differences compared to the spectrum of as-received a-C:H:Si:O. Namely, the $\mathrm{G}$ band is shifted towards higher wavenumbers, the ratio between the $\mathrm{D}$ and $\mathrm{G}$ band increase, and the photoluminescence background intensity increases.

These findings suggest that no significant structural changes occurred in a-C:H:Si:O upon sliding under either oxygen or hydrogen environment, in the volume probed by the Raman experiments (the optical mean free path of a-C:H:Si:O films has been reported by Scharf $e t a l .{ }^{24}$ to be $245 \pm 26 \mathrm{~nm}$ ). In contrast, the Raman analysis of the wear particles and the tribofilms formed on steel pins clearly indicate the occurrence of a structural reorganisation of the $\mathrm{sp}^{2}$ carbon phase, forming a material with $\mathrm{sp}^{2}$-hybridized carbon organized in clusters to a greater extent than in the as-grown film.

(a)

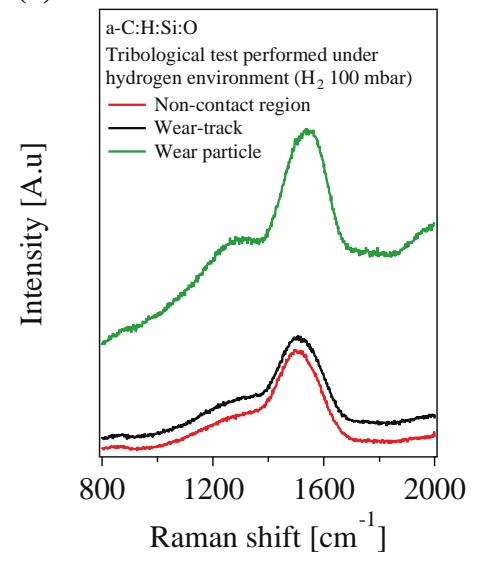

(b)

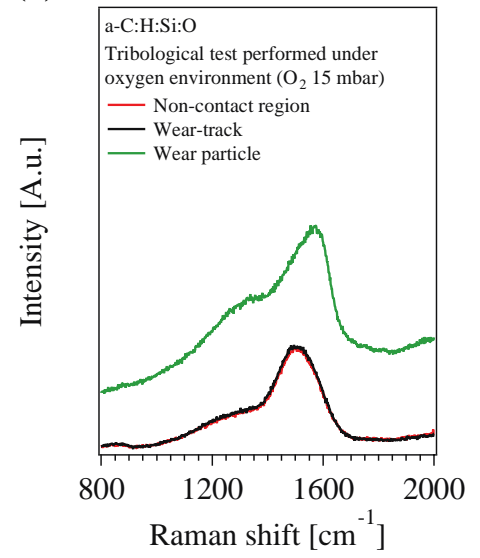

Figure.3. Raman spectra acquired on the unworn and worn region of a-C:H:Si:O as well as on the wear particle upon sliding against steel under 100 mbar of hydrogen (a) and 15 mbar of oxygen (b) 
To further investigate the structural transformations and surface reactions occurring on a-C:H:Si:O upon sliding under different environmental conditions, imaging near edge X-ray absorption fine structure spectroscopy (NEXAFS) measurements, which are highly surface sensitive (for electron yield NEXAFS spectroscopy of low atomic number elements, the information depth, which is the specimen thickness measured normal to the surface from which a specified percentage of typically $95 \%$ of the detected signal originates, is usually less than $5 \mathrm{~nm}^{25}$ ), were performed on a-C:H:Si:O samples with wear tracks formed under $\mathrm{HV}$ conditions, 15 mbar of oxygen and 100 mbar of hydrogen (note: two different samples were used for NEXAFS and SEM observation to avoid carbon contamination from electron beam exposure in SEM, and X-ray induced sample modification in NEXAFS). A typical carbon Kedge NEXAFS image is reported in the Supplementary Material (Figure S.1a).

The carbon K-edge spectrum extracted from the unworn region of a-C:H:Si:O (displayed in Figure 4 (inset). A larger plot of the NEXAFS spectra extracted from the corresponding C K-edge image is reported in the Supplementary Material, Figure S.1b) shows characteristic absorption features at $285.0 \pm 0.1 \mathrm{eV}$ (due to the $\mathrm{C} 1 \mathrm{~s} \rightarrow \pi^{*}$ transition for disordered carbon-carbon bonds), $\sim 286.5 \mathrm{eV}$ (due to the $\mathrm{C} 1 \mathrm{~s} \rightarrow \pi^{*}$ transition for carbonyl groups), $287.5 \pm 0.1 \mathrm{eV}$ (assigned to the $\mathrm{C} 1 \mathrm{~s} \rightarrow \sigma^{*}$ transition for carbon-hydrogen bonds), and $288.9 \pm 0.1 \mathrm{eV}$ (assigned to the $\mathrm{C} 1 \mathrm{~s} \rightarrow \sigma^{*}$ transition for carbon-oxygen and carbon-silicon bonds, as well as to the $\mathrm{C} 1 \mathrm{~s} \rightarrow \pi^{*}$ transition for carboxyl groups $)^{25,26}$. These assignments are summarized in Table 1.

Table.1. Summary of the carbon K-edge characteristic absorption features and the corresponding assignments

\begin{tabular}{cl}
\hline Peak Position $(\boldsymbol{e V})$ & Assignment \\
\hline $285.0 \pm 0.1$ & $\mathrm{C} 1 \mathrm{~s} \rightarrow \pi^{*}$ for disordered $\mathrm{C}=\mathrm{C}$ bonds \\
$\sim 286.5$ & $\mathrm{C} 1 \mathrm{~s} \rightarrow \pi^{*}$ transition for $\mathrm{C}=\mathrm{O}$ groups \\
$287.5 \pm 0.1$ & $\mathrm{C} 1 \mathrm{~s} \rightarrow \sigma^{*}$ transition for $\mathrm{C}-\mathrm{H}$ bonds \\
$288.9 \pm 0.1$ & $\mathrm{C} 1 \mathrm{~s} \rightarrow \sigma^{*}$ transition for $\mathrm{C}-\mathrm{O}$ and $\mathrm{C}-\mathrm{Si}$ bonds; $\mathrm{C} 1 \mathrm{~s} \rightarrow \pi^{*}$ transition for $\mathrm{O}=\mathrm{C}-\mathrm{OH}$ \\
\hline
\end{tabular}

Similarly, NEXAFS spectra were extracted from the wear tracks. To compare the worn regions of a-C:H:Si:O with the unworn region, a pre- and post-edge normalization was performed (Figure 4, upper right inset. A larger plot of the NEXAFS spectra extracted from the corresponding C K-edge image is reported in the Supplementary Material, Figure S.1b). In this way, variations in spectral intensity only arise from chemical changes and are independent of the total carbon content. In addition, to more easily investigate the structural 
transformations occurring in the near-surface region of a-C:H:Si:O upon sliding, the difference between spectra obtained from the worn and unworn a-C:H:Si:O regions was calculated (Figure 4). Computing these difference spectra also allows the contribution of the carbonaceous contamination layer present on the sample surface due to its exposure to air to be eliminated (this is valid under the assumption that the adventitious contamination layer is laterally homogeneous in composition, density, and thickness across both worn and unworn regions, i.e., is due to ambient exposure after the tribotesting; this approach avoids the need for correcting the as-acquired spectra for the presence of an adventitious carbonaceous contamination using a reference sample ${ }^{27}$ ). Since significant transfer of material from the steel pin to the a-C:H:Si:O surface occurs under $\mathrm{HV}$ or low gas pressures, the difference spectrum cannot be employed to elucidate the transformations occurring in the material in these cases. As for the tests performed under higher pressures of oxygen and hydrogen, the intensity of the $\mathrm{C} 1 \mathrm{~s} \rightarrow \pi^{*}$ transition for disordered carbon-carbon bonds at $285.0 \mathrm{eV}$ significantly increased, thus demonstrating a significant conversion from $\mathrm{sp}^{3}$ - to $\mathrm{sp}^{2}$-bonded carbon. Furthermore, a decrease in the signal at $288.9 \pm 0.1 \mathrm{eV}$ (assigned to the $\mathrm{C} 1 \mathrm{~s} \rightarrow \sigma^{*}$ transitions for $\mathrm{C}-\mathrm{O}$ and $\mathrm{C}-\mathrm{Si}$ bonds, as well as to the $\mathrm{C} 1 \mathrm{~s} \rightarrow \pi^{*}$ transition for $\mathrm{O}=\mathrm{C}-\mathrm{OH}$ groups) was observed. Finally, an intense peak at $286.0 \mathrm{eV}$ (due to the $\mathrm{C} 1 \mathrm{~s} \rightarrow \pi^{*}$ transition for carbonyl groups $)^{25,28}$ was detected in the difference spectrum obtained from the track produced under oxygen environment. This peak is also detected (with much less intensity) from the track produced under hydrogen, which can be due to some residual oxygen in the vacuum chamber.

To quantify the change in the carbon hybridization state upon sliding, the relative variation of the fraction of threefold-coordinated carbon atoms was calculated as follows (Equation.1):

$\Delta f_{s p^{2}}=100 * \frac{f_{s p_{2} \text { worn region }}-f_{s p_{2}}{ }_{\text {unworn region }}}{f_{s p_{2} \text { unworn region }}}$

The results are displayed as a function of the entrance grid bias (EGB) voltage in the lower inset of Figure 4 (increasing the EGB voltage allows NEXAFS measurements with higher surface sensitivity to be performed ${ }^{21}$ ). Upon increasing the EGB voltage, a higher fraction of $\mathrm{sp}^{2}$-bonded carbon was detected independently of the gas environment. This finding suggests the formation of a gradient in carbon bonding configuration, with even more 
conversion to $\mathrm{sp}^{2}$-bonded carbon produced in the near-surface region of a-C:H:Si:O during sliding.

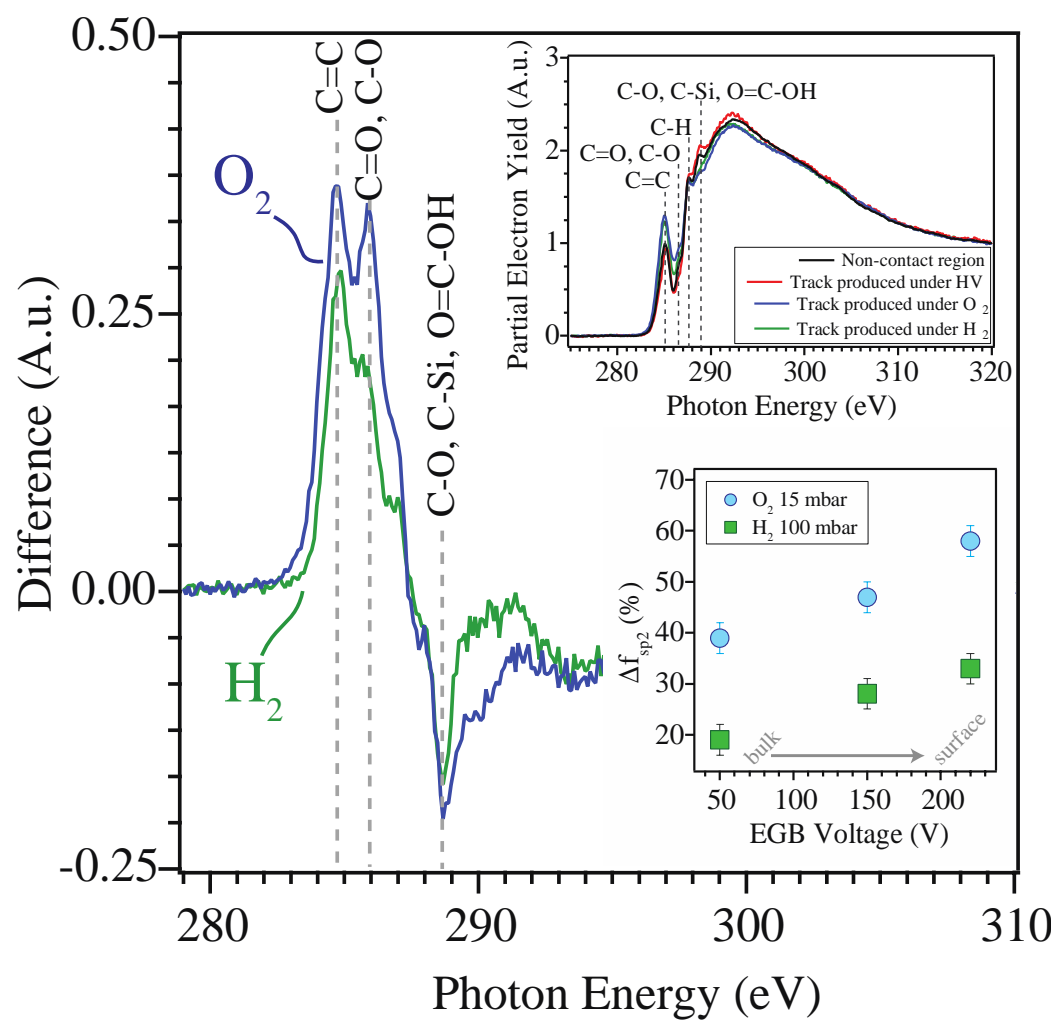

Figure 4. Difference in the NEXAFS partial electron yield spectra between worn and unworn regions of a-C:H:Si:O Green curve: difference between a spectrum taken from the wear track generated upon sliding in hydrogen $(100$ mbar $)$ and a spectrum from unworn a-C:H:Si:O; Blue curve: difference between a spectrum taken from the wear track generated upon sliding in oxygen (15 mbar) and a spectrum from unworn aC:H:Si:O. The upper inset shows the pre- and post-edge normalized spectra taken from worn (wear track generated under HV conditions or in oxygen and hydrogen environments) and unworn regions. The lower inset shows the relative variation of the fraction of $\mathrm{sp}^{2}$-bonded carbon for the track generated in hydrogen (100 mbar) or oxygen (15 mbar) as a function of the entrance grid bias (EGB) voltage (the surface sensitivity increases with the EGB voltage).

\section{Discussion}

\subsection{Abrasion vs. adhesion in the friction mechanism of a-C:H:Si:O}

The friction results presented in Section 3.1 reveal that the tribological behaviour of a$\mathrm{C}: \mathrm{H}: \mathrm{Si}$ :O films strongly depends on the sliding environment, i.e., the amount and nature of gas introduced in the vacuum chamber. Under HV or low gas pressure, the coefficient of 
friction increases rapidly and reaches 1.2 just after few sliding cycles (15). The transfer of iron from the steel pin to the a-C:H:Si:O film after tribotests under $\mathrm{HV}$ or low gas pressure, revealed by SEM/EDX analyses, indicates that strong adhesion occurred between a-C:H:Si:O and steel (i.e., formation of bonds across the sliding interfaces, in agreement with Gao et $\left.a l .{ }^{29}\right)$, followed by a break of the adhesive junctions at the steel side. The close hardness values of the steel pin and the a-C:H:Si:O (9 $\pm 1 \mathrm{GPa}$ and $10 \pm 1 \mathrm{GPa}$, respectively) and the relative smoothness of the surfaces $(1.3 \pm 0.1 \mathrm{~nm}$ and $27.3 \pm 0.2 \mathrm{~nm}$ for the a-C:H:Si:O coating and the steel pin respectively) indicate that this transfer phenomenon is a consequence of adhesion, rather than abrasion of the steel by the a-C:H:Si:O followed by an accumulation of the abrasive debris on a-C:H:Si:O film's surface. This is consistent with Fontaine et al. ${ }^{30}$, who showed that while sliding a very smooth a-C:H film against steel counterbody, adhesion occurs between the flat and the pin by starting at some key locations on the flat and spread to the whole wear track. Molecular dynamics simulations of such systems indeed find that the generation of covalent bonds through the interface induces an increase in friction upon sliding $^{31,32}$.

A relatively small pressure of oxygen or hydrogen gas is required to transition from the high friction regime to the relatively low friction regime: 10 mbar of oxygen gas or 50 mbar of hydrogen gas. This indicates that the reduction of friction is due to tribochemical reactions, which require a sufficiently abundant source of gas molecules to achieve and maintain low friction.

In addition, at these intermediate and higher gas pressures (>10 mbar oxygen, >50 mbar hydrogen) there is no more transfer from the steel pin to the film as observed under HV. Rather, there is transfer in the other direction: from the a-C:H:Si:O to the steel pin. The gas thus plays a key role in determining the friction response by inducing tribochemical reactions that prevent the mechanical transfer of steel to a-C:H:Si:O film, observed under HV. In light of this, the transfer phenomena observed in the experiments can be interpreted as a consequence of the creation of adhesive junctions between the two surfaces and not because of abrasion.

\subsection{Surface passivation vs. rehybridization in determining the friction response of a- C:H:Si:O}

NEXAFS spectra (Figure 4) acquired inside the worn region after tribotests either under oxygen or hydrogen revealed the presence of an $\mathrm{sp}^{2}$-bonded carbon rich surface layer, with an increasing $\mathrm{sp}^{2}$ concentration towards the surface. This is in agreement with Le Huu et 
al., who have shown a transformation of $\mathrm{sp}^{3}$ - to $\mathrm{sp}^{2}$-bonded carbon upon sliding. This rehybridization phenomenon is also supported by experimental data from self-mated sliding studies of diamond and tetrahedral amorphous carbon (ta-C) films ${ }^{33,34}$ and molecular dynamics simulations of sliding of hydrogenated and hydrogen-free amorphous carbon films ${ }^{32,35}$.

In addition, according to NEXAFS analysis, the wear track produced when sliding in oxygen environment exhibited carbon-oxygen bonds in the near-surface region of a$\mathrm{C}: \mathrm{H}: \mathrm{Si}$ :O. This increase in intensity of the signal assigned to carbon-oxygen bonds (286.0 $\mathrm{eV}$ ) could be interpreted as the dissociative reaction of oxygen molecules with strained $\mathrm{sp}^{2}-$ hybridized $\mathrm{C}-\mathrm{C}$ bonds, which are produced upon sliding ${ }^{35}$. This reaction results in the formation of an oxygen-passivated layer on a highly $\mathrm{sp}^{2}$-bonded carbon surface produced by sliding on a-C:H:Si:O in oxygen.

However, according to the NEXAFS analysis, the wear track produced in hydrogen environment did not lead to a significant increase in $\mathrm{C}-\mathrm{H}$ bonds at the surface. In addition, the near-surface region of this track exhibits much less $\mathrm{sp}^{2}$-bonded carbon than the wear track produced under oxygen environment (note: the tribological conditions used for performing experiments were the same for the different environments). This suggests that either hydrogen is etching the newly generated $\mathrm{sp}^{2}$ strained carbon layer, or the carbon atoms react with hydrogen to generate volatile hydrocarbon fragments. This finding is different than results reported by Fontaine et al. ${ }^{36}$, Donnet et $a .^{37}$, and Erdemir et al. ${ }^{38}$ who studied hydrogenated amorphous carbon films under HV and in presence of hydrogen or in dry environment. Matta et al. ${ }^{39}$, also studied tetrahedral hydrogen free amorphous carbon films in presence of $\mathrm{OH}$-containing environments. They all explained the decrease of the friction in the presence of hydrogen or $\mathrm{O}-\mathrm{H}$ elements respectively, as a consequence of the passivation of the $s p^{2}$ bonded carbon by either hydrogen of $\mathrm{O}-\mathrm{H}$ bonds.

This result motivates our new model below to explain the tribochemical reactions occurring for a-C:H:Si:O sliding against steel under HV and controlled pressures of oxygen and hydrogen gas.

\subsection{Friction model for a-C:H:Si:O under $H V$ and in presence of oxygen and hydrogen.}

On the basis of the findings mentioned above, the model depicted in Scheme 1 is suggested for explaining the friction response of a-C:H:Si:O under different environmental conditions.

Stage 1: Initial state without applying any mechanical stress; 
> Stage 2: Upon sliding, asperities come into contact. As shown by several authors, ${ }^{35,40}$ rehybridization phenomenon (stress-induced conversion from $\mathrm{sp}^{3}$ - to $\mathrm{sp}^{2}$-bonded carbon) occurs at the contact area. The initial and newly-generated $\mathrm{sp}^{2}$-bonded carbon is highly strained and thus prone to react with the environment ${ }^{39}$;

$>$ Stage 3, under HV and low gas pressure: Upon further sliding, the reactive $\mathrm{sp}^{2}$ bonded carbon atoms at the surface interact with the steel counterbody and create adhesive junctions. The interaction of carbon $\mathrm{sp}^{2}$ orbitals under $\mathrm{HV}$ has been also observed by Donnet et al. ${ }^{37}$;

$>$ Stage 4, under HV and low gas pressure: Some adhesive bonds are stronger than the metallic bonds in the steel countersurface, thus leading to a transfer of steel onto the a-C:H:Si:O. The large amount of energy required to break metallic bonds in steel results in high friction under HV conditions.

> Stage 3', under intermediate and high gas pressure: The observed transfer of material from the coating to the steel pin indicates that interfacial adhesion, as described at Stage 3 (under HV and low gas pressure), still occurs. However, the direction of the material transfer is changed: carbon, silicon, and oxygen are observed transferred to the steel countersurface (hydrogen is presumed to be transferred as well), whereas no iron is transferred to a-C:H:Si:O. Based on our NEXAFS data, a layer with a higher fraction of $\mathrm{sp}^{2}$-hybridized carbon is formed during sliding, similar to simulations and experiments for self-mated sliding of diamond and ta-C films. ${ }^{34,35,41}$ In the presence of highly reactive gases, the strained $\mathrm{sp}^{2}$ carbon-carbon bonds can easily break, thus leading to transfer of material to the steel countersurface and leaving behind a passivated highly-sp ${ }^{2}$-bonded carbon surface. The increase of $\mathrm{C}=\mathrm{O}$ peak intensity at $286.0 \mathrm{eV}$ in the track obtained upon sliding under oxygen supports this. The fact that the C-H peak does not increase in intensity for sliding in hydrogen environments may be due to the highly favourable reaction of hydrogen molecules with strained $\mathrm{sp}^{2}$ carbon-carbon bonds, resulting in either an easier removal of material from the wear track or the formation of volatile short-chain hydrocarbon fragments. More work is required to have insights into the dominant tribochemical reaction(s). The etching of the softer, disordered, highly-sp ${ }^{2}$-bonded carbon layer is supported by the fact that the absolute values of the variation in the fraction of $\mathrm{sp}^{2}$ bonded carbon in the near-surface region of the wear track created upon sliding under hydrogen environment is lower than upon sliding in oxygen (lower inset, Figure 4). This idea is also supported by the fact that the wear volume of the a-C:H:Si:O is 
higher in hydrogen than oxygen environments: $(1.2 \pm 0.2) 10^{-6} \mathrm{~mm}^{3} \mathrm{~N}^{-1} \mathrm{~m}^{-1}$ and (5.7 \pm 0.2$) 10^{-7} \mathrm{~mm}^{3} \mathrm{~N}^{-1} \mathrm{~m}^{-1}$ respectively (see Supplementary Material, Figure S.2);

Stage 4', under intermediate and high gas pressure: Under the combined effect of a larger total sliding distance of the steel pin (higher than the worn region of the a$\mathrm{C}: \mathrm{H}: \mathrm{Si}: \mathrm{O}$ flat) and the applied load and stress, the transferred $\mathrm{sp}^{2}$ hybridized carbonrich top layer on the steel pin, has enough energy to reorganize itself into larger aromatic clusters, leading then to a $\mathrm{sp}^{2}$ layer rich in ring-like structures between the steel pin and the flat, as indicated by our Raman measurements. Nevertheless, once the modified carbon tribofilm is generated in the contact, the friction will no longer only depend on the way the adhesive junctions are released (the energy that is needed to break the bonds in the steel side or in the a-C:H:Si:O) but also on the shear strength of this tribofilm. In fact, as shown by Scharf et al., ${ }^{16}$ the shear strength of tribofilms generated when sliding against a-C:H:Si:O depends on its chemical composition, which is a function of the sliding environment. This might explain the small but observable differences in the friction coefficients obtained under oxygen gas or hydrogen gas.
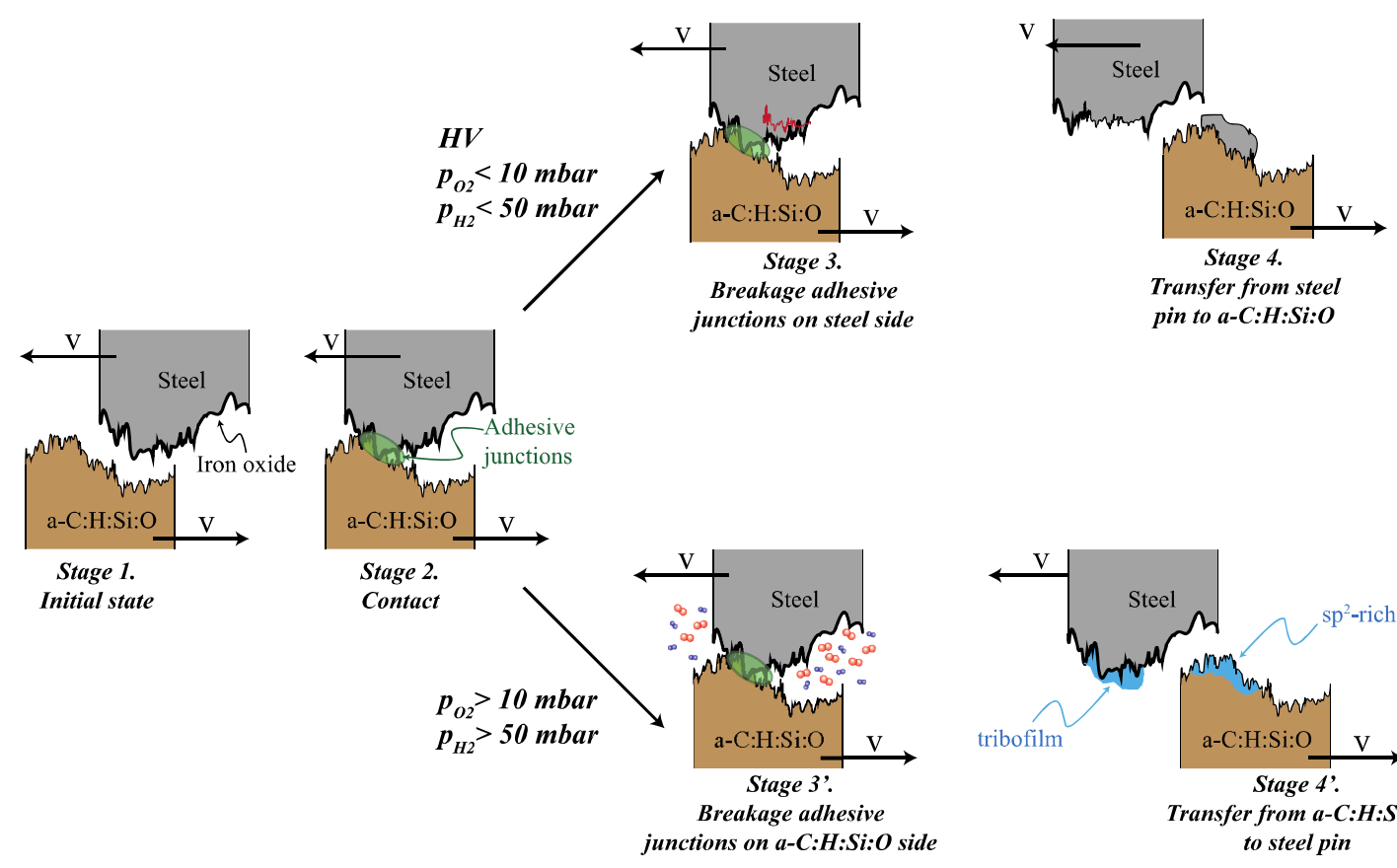

Figure 5. Schematic model for describing the friction properties of an a-C:H:Si:O vs. steel tribosystem sliding under different environmental conditions. 


\section{Conclusions}

The tribological response of a-C:H:Si:O film was investigated under different environmental conditions, ranging from high vacuum to elevated oxygen and hydrogen pressures. Three major conclusions can be drawn on the basis of the results presented in this work:

1. By using an environment-controlled tribometer from high vacuum up to one atmosphere, we were able to determine, for the first time, a threshold in oxygen (10 mbar) or hydrogen gas pressure (50 mbar), necessary to switch from a high friction regime to a low friction regime. This indicates that the reduction of friction is due to tribochemical reactions, which require a sufficiently abundant source of gas molecules to achieve and maintain low friction;

2. Under high vacuum and low gas pressure $(\mathrm{p}<10$ mbar of oxygen or $\mathrm{p}<50$ mbar of hydrogen), transfer of material from the steel pin to the a-C:H:Si:O flat was observed. In contrast, above 10 mbar of oxygen and 50 mbar hydrogen, transfer of material takes place from the a-C:H:Si:O flat to the steel pin, thus building a tribofilm on the steel countersurface. This latter tribofilm has a low shear strength;

3. The NEXAFS characterization of a-C:H:Si:O after the tribological tests revealed that upon sliding under oxygen ( $p>10$ mbar) or hydrogen ( $p>50$ mbar) environments, a surface layer with a higher fraction of $\mathrm{sp}^{2}$-bonded carbon atoms compared to the unworn material is formed on a-C:H:Si:O.

The outcomes of the present investigation suggest that the build-up and release of adhesive junctions between steel and a-C:H:Si:O strongly depends on the environment. In particular, the tribochemical changes occurring in a-C:H:Si:O upon sliding in the presence of oxygen ( $p$ $>10$ mbar) or hydrogen ( $p>50$ mbar) indicate, for the first time, that these gases favor the release of the chemical bonds by dissociatively reacting with the mechanically-stressed $\mathrm{sp}^{2}$ carbon-rich surface layer in the near surface of a-C:H:Si:O. 


\section{Acknowledgements}

This material is based upon work supported by the National Science Foundation under Grant No. DMR-1107642 and by the Agence Nationale de la Recherche under grant No. ANR-11NS09-01 through the Materials World Network program. F.M. acknowledges support from the Marie Curie International Outgoing Fellowship for Career Development within the $7^{\text {th }}$ European Community Framework Program under contract no. PIOF-GA-2012-328776. K.K acknowledges Région Rhône-Alpes and University de Lyon for their fellowship support through CMIRA and PALSE program respectively. J.B.M., F.M. and R.W.C. acknowledge support from the Advanced Storage Technology Consortium ASTC (grant 2011-012). The authors would like to thank Dr. C. Jaye and Dr. D.A. Fischer for the kind assistance with the NEXAFS measurements at the National Synchrotron Light Source. Use of the National Synchrotron Light Source, Brookhaven National Laboratory, was supported by the US Department of Energy, Office of Science, and Office of Basic Energy Sciences, under Contract No. DE-AC02-98CH10886. We acknowledge Th. Le Mogne and M. Guibert for their technical support with the environment-controlled tribometer and CLYM (Centre Lyonnais de Microscopie) for the use of the Scanning Electron Microscope. 


\section{References}

1. Christophe Donnet - Ali Erdemir. Tribology of Diamond-Like Carbon Films. (2008).

2. Prasad, S. V., Dugger, M. T., Christenson, T. R. \& Tallant, D. R. LIGA Microsystems: Surface Interactions, Tribology, and Coatings. J. Manuf. Process. 6, 107-116 (2004).

3. Grill, A. Diamond-like carbon coatings as biocompatible materials-an overview. Diam. Relat. Mater. 12, 166-170 (2003).

4. Hauert, R. A review of modified DLC coatings for biological applications. Diam. Relat. Mater. 12, 583-589 (2003).

5. Sullivan, J. P., Friedmann, T. A. \& Baca, A. G. Stress relaxation and thermal evolution of film properties in amorphous carbon. J. Electron. Mater. 26, 1021-1029 (1997).

6. Sánchez-López, J. C. \& Fernández, A. in Tribology of Diamond-Like Carbon Films (eds. Donnet, C. \& Erdemir, A.) 311-338 (Springer US, 2008). at <http://link.springer.com/chapter/10.1007/978-0-387-49891-1_12>

7. Tripathi, R. K., Panwar, O. S., Srivastava, A. K., Rawal, I. \& Chockalingam, S. Structural, nanomechanical, field emission and ammonia gas sensing properties of nitrogenated amorphous carbon films deposited by filtered anodic jet carbon arc technique. Talanta 125, 276-283 (2014).

8. Panwar, O. S. et al. Effect of high substrate bias and hydrogen and nitrogen incorporation on spectroscopic ellipsometric and atomic force microscopic studies of tetrahedral amorphous carbon films. Surf. Coat. Technol. 205, 2126-2133 (2010).

9. Panwar, O. S. et al. Characterization of Boron- and Phosphorous-Incorporated Tetrahedral Amorphous Carbon Films Deposited by the Filtered Cathodic Vacuum Arc Process. Jpn. J. Appl. Phys. 48, 065501 (2009).

10. Neerinck, D. et al. Diamond-like nanocomposite coatings for low-wear and low-friction applications in humid environments. Thin Solid Films 317, 402-404 (1998).

11. Neerinck, D. et al. Diamond-like nanocomposite coatings (a-C:H/a-Si:O) for tribological applications. Diam. Relat. Mater. 7, 468-471 (1998).

12. Mangolini, F., Rose, F., Hilbert, J. \& Carpick, R. W. Thermally induced evolution of hydrogenated amorphous carbon. Appl. Phys. Lett. 103, 161605 (2013).

13. K. Oguri and T. Arai. Low friction coatings of diamond-like carbon with silicon prepared by plasma-assisted chemical vapor deposition. J Mater Res Vol 5 No 11 (1990).

14. K. Oguri and T. Arai. Two different low friction mechanisms of diamond-like carbon with silicon coatings formed by plasma-assisted chemical vapor deposition. J Mater Res Vol (1992).

15. Gilmore, R. \& Hauert, R. Control of the tribological moisture sensitivity of diamond-like carbon films by alloying with F, Ti or Si. Thin Solid Films 398-399, 199-204 (2001).

16. Scharf, T. W., Ohlhausen, J. A., Tallant, D. R. \& Prasad, S. V. Mechanisms of friction in diamondlike nanocomposite coatings. J. Appl. Phys. 101, 063521-063521-11 (2007).

17. Kester, D. J., Brodbeck, C. L., Singer, I. L. \& Kyriakopoulos, A. Sliding wear behavior of diamond-like nanocomposite coatings. Surf. Coat. Technol. 113, 268-273 (1999).

18. Venkatraman, C., Brodbeck, C. \& Lei, R. Tribological properties of diamond-like nanocomposite coatings at high temperatures. Surf. Coat. Technol. 115, 215-221 (1999).

19. Tannous, J., de Bouchet, B. M. I., Le-Mogne, T., Charles, P. \& Martin, J. M. Contribution of gas phase lubrication in understanding tribochemistry of organosulphur compounds. Tribol. - Mater. Surf. Interfaces 1, 98-104 (2007).

20. Konicek, A. R. et al. Near-Edge X-ray Absorption Fine Structure Imaging of Spherical and Flat Counterfaces of Ultrananocrystalline Diamond Tribological Contacts: A Correlation of Surface Chemistry and Friction. Tribol. Lett. 44, 99-106 (2011).

21. Genzer, J., Kramer, E. J. \& Fischer, D. A. Accounting for Auger yield energy loss for improved determination of molecular orientation using soft x-ray absorption spectroscopy. J. Appl. Phys. 92, 7070-7079 (2002).

22. Ferrari, A. C. \& Robertson, J. Interpretation of Raman spectra of disordered and amorphous carbon. Phys. Rev. B 61, 14095-14107 (2000).

23. Rose, F. et al. Complete characterization by Raman spectroscopy of the structural properties of thin hydrogenated diamond-like carbon films exposed to rapid thermal annealing. J. Appl. Phys. 116, 123516 (2014). 
24. Scharf, T. W. \& Singer, I. L. Thickness of diamond-like carbon coatings quantified with Raman spectroscopy. Thin Solid Films 440, 138-144 (2003).

25. Stöhr, J. NEXAFS Spectroscopy. (Springer Science \& Business Media, 1992).

26. Robin, M. B., Ishii, I., McLaren, R. \& Hitchcock, A. P. Fluorination effects on the inner-shell spectra of unsaturated molecules. J. Electron Spectrosc. Relat. Phenom. 47, 53-92 (1988).

27. Mangolini, F., McClimon, J. B., Rose, F. \& Carpick, R. W. Accounting for Nanometer-Thick Adventitious Carbon Contamination in X-ray Absorption Spectra of Carbon-Based Materials. Anal. Chem. (2014). doi:10.1021/ac503409c

28. Ishii, I. \& Hitchcook, A. P. The oscillator strengths for $\mathrm{C} 1 \mathrm{~s}$ and $\mathrm{O} 1 \mathrm{~s}$ excitation of some saturated and unsaturated organic alcohols, acids and esters. J. Electron Spectrosc. Relat. Phenom. 46, 5584 (1988).

29. Gao, G. T., Mikulski, P. T. \& Harrison, J. A. Molecular-Scale Tribology of Amorphous Carbon Coatings: Effects of Film Thickness, Adhesion, and Long-Range Interactions. J. Am. Chem. Soc. 124, 7202-7209 (2002).

30. Fontaine, J., Belin, M., Le Mogne, T. \& Grill, A. How to restore superlow friction of DLC: the healing effect of hydrogen gas. Tribol. Int. 37, 869-877 (2004).

31. Gao, G. T., Mikulski, P. T. \& Harrison, J. A. Molecular-scale tribology of amorphous carbon coatings: effects of film thickness, adhesion, and long-range interactions. J. Am. Chem. Soc. 124, 7202-7209 (2002).

32. Schall, J. D., Gao, G. \& Harrison, J. A. Effects of Adhesion and Transfer Film Formation on the Tribology of Self-Mated DLC Contacts. J. Phys. Chem. C 114, 5321-5330 (2010).

33. Konicek, A. R. et al. Influence of surface passivation on the friction and wear behavior of ultrananocrystalline diamond and tetrahedral amorphous carbon thin films. Phys. Rev. B 85, 155448 (2012).

34. Konicek, A. R. et al. Origin of Ultralow Friction and Wear in Ultrananocrystalline Diamond. Phys. Rev. Lett. 100, 235502 (2008).

35. Kunze, T. et al. Wear, Plasticity, and Rehybridization in Tetrahedral Amorphous Carbon. Tribol. Lett. 53, 119-126 (2014).

36. Fontaine, J., Le Mogne, T., Loubet, J. L. \& Belin, M. Achieving superlow friction with hydrogenated amorphous carbon: some key requirements. Thin Solid Films 482, 99-108 (2005).

37. Donnet, C., Fontaine, J., Grill, A. \& Mogne, T. L. The role of hydrogen on the friction mechanism of diamond-like carbon films. Tribol. Lett. 9, 137-142 (2001).

38. Erdemir, A. The role of hydrogen in tribological properties of diamond-like carbon films. Surf. Coat. Technol. 146-147, 292-297 (2001).

39. Matta, C. et al. Tribochemistry of tetrahedral hydrogen-free amorphous carbon coatings in the presence of OH-containing lubricants. Lubr. Sci. 20, 137-149 (2008).

40. Le Huu, T., Zaidi, H., Paulmier, D. \& Voumard, P. Transformation of sp3 to sp2 sites of diamond like carbon coatings during friction in vacuum and under water vapour environment. Thin Solid Films 290-291, 126-130 (1996).

41. Konicek, A. R. et al. Influence of surface passivation on the friction and wear behavior of ultrananocrystalline diamond and tetrahedral amorphous carbon thin films. Phys. Rev. B 85, 155448 (2012). 